\title{
Dynamic Buckling of a FGM Euler-Bernoulli Beam under Thermal Shock via Symplectic Method Jing-Hua ZHANG ${ }^{1, a}$, Xing-Xing $\mathrm{ZHAO}^{1, \mathrm{~b},{ }^{*}}$ \\ ${ }^{1}$ School of Sciences, Lanzhou University of Technology, Lanzhou 730050, China \\ azjhhrb@163.com, 'bhaoxx0810@126.com \\ ${ }^{*}$ Corresponding author
}

Keywords: Euler-Bernoulli Beam, FGMs, Thermal Shock, Symplectic Method, Dynamic Buckling.

\begin{abstract}
Based on Euler-Bernoulli beam theory, the dynamic buckling of a functionally graded beam subjected to thermal shock is investigated in the Symplectic space. The boundaries of the FGM beam are simply supported at one end, and the other is clamped. The dynamic buckling problem is reduced to zero-eigenvalue problem. The buckling mode and critical thermal axial forces can be obtained through bifurcation condition, and the critical temperature rise of the functionally graded beam is obtained by inverse solution.
\end{abstract}

\section{Introduction}

Functionally graded materials (FGMs) have been regarded as one of the advanced inhomogeneous composite materials. The structure made from functionally graded materials, often operates in high temperature gradient thermal environment with static or dynamic thermal loads. Buckling and post-buckling caused by thermal loads have been attracted much research interest of mechanics workers [1].

Up to now, most of the research on functionally graded structural thermal buckling or thermal post-buckling is limited to static problems. Javaheri and Eslami[2] investigated the thermal buckling of rectangular FGM plate based on classical as well as higher-order shear deformation theories and obtained the closed form solutions of the problem under several types of thermal loadings. Based on the first-order and the third-order shear deformation theories, investigation of nonlinear bending and post-buckling of circular functionally graded plates subjected to mechanical and thermal loadings were performed by Ma and Wang [3].

In contrast with the static buckling, dynamic buckling of functionally graded structures under thermal shock is much less. Based on the classical shell theory, dynamic thermal post-buckling behavior of functionally graded cylindrical shells subjected to the combined action of thermal load and applied actuator voltage was analyzed by Mirzavand, etc. [4], in which the temperature dependency of FGM properties was considered. Then, a finite difference method combined with the Runge-Kutta method was employed to predict the post-buckling equilibrium paths, and the dynamic buckling temperature difference was detected according to Budiansky's stability criterion. On the basis of the obtained results, Mirzavand etc. [5] predicted post-buckling equilibrium path and obtained dynamic buckling temperature of piezoelectric FGM cylindrical shell, based on the third-order shear deformation shell theory. Dynamic thermal buckling of suddenly heated FGM cylindrical shells under combined axial compression and external pressure was also investigated by Shariyat[6] using the finite element method, where the temperature dependency of FGM properties is considered.

However, the traditional methods were adopted to solve elastic mechanics in aforementioned studies. Herein, the Symplectic method is used to study the stability of functionally graded Euler-Bernoulli beam under thermal shock. 


\section{Problem Formulations}

\section{Material Properties of the FGM Beam}

We consider a FGM beam (length $l$, thickness $h$ and width $b$ ) with rectangular cross-section, which is made from a mixture of ceramics and metals. The material properties of the FGM beam are assumed to be varied only in the thickness direction from full metal at the top surface to full ceramic at the bottom. The volume fractions are expressed by a simple power law distribution, as follows.

$$
P(z)=\left(P_{c}-P_{m}\right) V_{c}(z)+P_{m}, V_{c}(z)=\left(\frac{h-2 z}{2 h}\right)^{k}
$$

Where $P_{c}$ and $P_{m}$ are material properties of ceramic and metal, respectively. $V_{c}(z)$ is the volume content of the ceramic, $k$ is the volume fraction index of the ceramic.

\section{Canonical Equations}

The corresponding displacements in any point of the beam are designated $u(x, t)$ and $w(x, t)$ in directions of axis $\mathrm{x}$ and transverse $\mathrm{z}$, respectively. FGMs are considered as a linear thermo-elastic. According to Euler-Bernoulli beam theory, the axial stress $\sigma_{x}$ can be expressed by

$$
\sigma_{x}=E(z)\left[\frac{\partial u}{\partial x}-z \frac{\partial^{2} w}{\partial x^{2}}-\alpha(z) T(z, t)\right] .
$$

Where $T(z, t)$ (Unit: $\mathrm{K})$ is the temperature variations, $t$ (Unit: $\mathrm{s}$ ) is the time.

The FGM beam is considered as one end fixed and other end simply supported, the boundary conditions are expressed by

$$
x=0, u=w=0, \frac{\partial w}{\partial x}=0 ; x=l, u=w=0, \frac{\partial^{2} w}{\partial x^{2}}=0 .
$$

The bending deformation can play a dominated role in all the deformations of the beam. For FGM beams, Lagrange function can be expressed by

$$
L=\frac{1}{2} I\left(\frac{\partial w}{\partial t}\right)^{2}-D \kappa^{2}-\frac{1}{2} N^{T}\left(\frac{\partial w}{\partial x}\right)^{2}-\frac{1}{2} M^{T} \kappa .
$$

Where $I$ is the quality of unit length of FGM beam, $D$ is stiffness coefficient of FGM beam, $N^{T}$ and $M^{T}$ are thermal axial force and thermal moment, respectively. $I, D, N^{T}$ and $M^{T}$ are defined as

$$
I=b \int_{-h / 2}^{h / 2} \rho(z) \mathrm{d} z, D=b \int_{-h / 2}^{h / 2} E(z) \mathrm{z}^{2} \mathrm{~d} z,\left(N^{T}, M^{T}\right)=b \int_{-h / 2}^{h / 2} E(z) \alpha(z) T(z, t)(1, z) \mathrm{d} z .
$$

For introducing the Hamilton System, the original variable are defined by $w=q, \dot{f}=\frac{\partial f}{\partial t}$, where $f$ is a random variable. The dual variables is defined by

$$
p=\frac{\delta L}{\delta \dot{q}}=I \dot{q} .
$$

Where $\delta$ denotes the variation, ${ }^{p}$ represents the momentum of system. $\mathrm{H}$ is a Hamilton function of system. It can be expressed by 


$$
H=p \dot{q}-L=\frac{p^{2}}{2 I}+\frac{D}{2}\left(\frac{\partial^{2} q}{\partial x^{2}}\right)^{2}+\frac{1}{2} N^{T}\left(\frac{\partial q}{\partial x}\right)^{2}+\frac{1}{2} M^{T} \frac{\partial^{2} q}{\partial x^{2}}
$$

According to Hamilton principle, dual canonical equations in the Hamilton system can be obtained:

$$
\dot{\Psi} \equiv\left\{\begin{array}{l}
\dot{q} \\
\dot{p}
\end{array}\right\}=\left\{\begin{array}{l}
\frac{\delta H}{\delta p} \\
-\frac{\delta H}{\delta q}
\end{array}\right\}=\left\{\begin{array}{l}
\frac{p}{I} \\
-D \frac{\partial^{4} q}{\partial x^{4}}-N^{T} \frac{\partial^{2} q}{\partial x^{2}}
\end{array}\right\} .
$$

Then, in the Hamilton system, the boundary conditions (3) can be expressed by

$$
x=0, q=0, \frac{\partial q}{\partial x}=0 ; x=l, q=0, \frac{\partial^{2} q}{\partial x^{2}}=0 \text {. }
$$

\section{The Transient Temperature Field}

The dynamic response of FGM beam under the initial steady-state heat balance environment and suddenly subjected to uniform thermal loads on its bottom surface is investigated. The temperature field of the beam varies with time and the location of the thickness direction, and its upper surface exchanges heat with the external environment. Thus, the heat conduction equation in the absence of internal heat sources reduces to

$$
C(z) \rho(z) \frac{\partial T}{\partial t}=\frac{\partial}{\partial z}\left\lfloor K(z) \frac{\partial T}{\partial z}\right\rfloor,\left(t>0,-\frac{h}{2}<z<\frac{h}{2}\right) .
$$

The thermal initial conditions and the boundary conditions on the lower and the upper surfaces, are specified as

$$
T(z, 0)=0, T\left(-\frac{h}{2}, t\right)=\Delta T\left(1-e^{-a t}\right),-\left.K(z) \frac{\partial T}{\partial z}\right|_{z=h / 2}=h_{r} T\left(\frac{h}{2}, t\right) .
$$

Where $^{h_{r}}$ is the heat exchange coefficient between the upper surface of beam and the environment. Here, the Laplace transformation technique and the power series method are used to solve Eqs. $(10,11)$, and the transient temperature field in the FGM beam can be obtained.

\section{Solving the Canonical Equations}

Introduce the following dimensionless quantities for an easy solution.

$$
X=\frac{x}{l}, Q=\frac{q}{l}, \quad \theta=\frac{N^{T} l^{2}}{D}
$$

In the Hamilton system, a dimensionless form of the canonical equation is expressed by

$$
\frac{\partial^{4} Q}{\partial X^{4}}+\theta \frac{\partial^{2} Q}{\partial X^{2}}=0
$$

Equation (13) is a linear homogeneous equations, its general solution is given by

$$
Q=C_{1}+C_{2} X+C_{3} \cos (\sqrt{\theta} X)+C_{4} \sin (\sqrt{\theta} X) \text {. }
$$


Substituting the boundary Conditions into Equation (14) and solving it, we can obtain bifurcation conditions and buckling modes equation, as follows:

$$
-(\theta+1) \cos \sqrt{\theta}+\sqrt{\theta} \sin \sqrt{\theta}+1=0, Q_{n}=\tan \sqrt{\theta}\lfloor 1-\cos (\sqrt{\theta} X)\rfloor+\lfloor\sin (\sqrt{\theta} X)-\sqrt{\theta} X\rfloor
$$

\section{Numerical Results and Discussions}

The bifurcation condition of functionally graded beams is solved by Newton-Raphson method. So, the dimensionless characteristic values can be obtained, there are

$\theta_{1}=20.19, \theta_{2}=59.67, \theta_{3}=118.90, \theta_{4}=197.85, \theta_{5}=296.55$,

Then, substituting $\theta_{n}$ into equation (15), the buckling mode can be obtained. First and second buckling modes are plotted in Fig.1 and Fig.2.

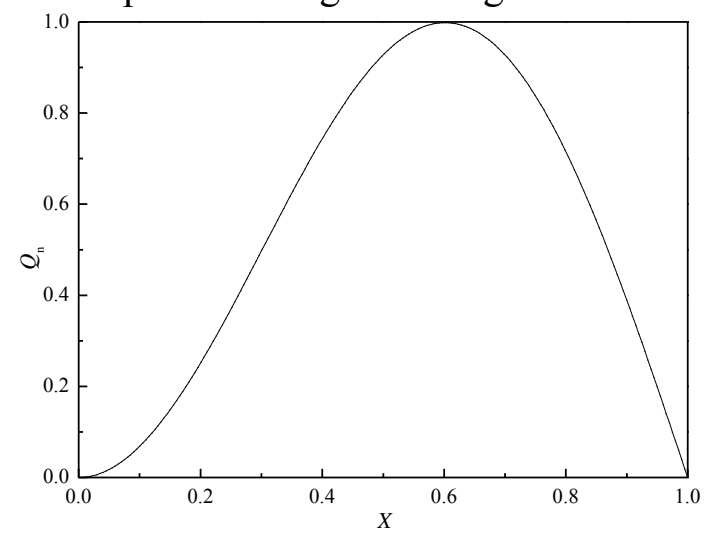

Fig.1 First Order Critical Buckling Mode

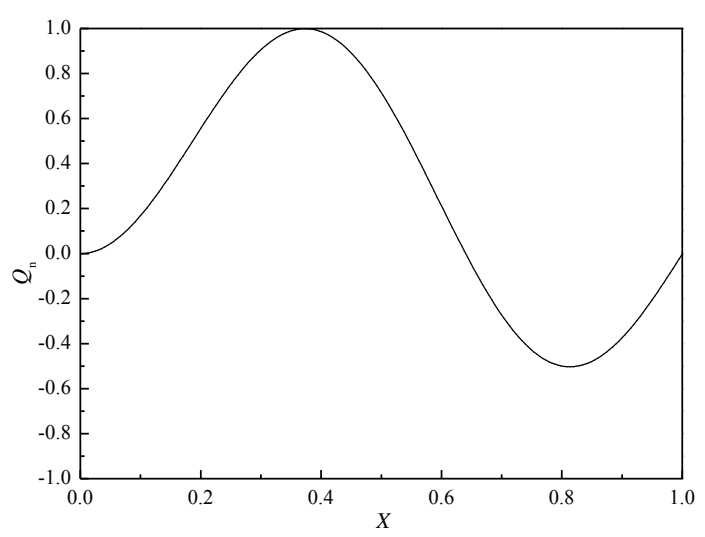

Fig. 2 Second Order Critical Buckling Mode

In the computation, the FGM beam made from $\mathrm{SiC}$ and $\mathrm{Ni}$ is considered. Material properties of the constituents can be found in reference [7]. If no special specification, the geometries of the FGM beam are $l=0.4 \mathrm{~m}, h=0.01 \mathrm{~m}, \Delta t=5 \mathrm{~s}$ and $a=10$. The heat exchange coefficient is $h_{r}=50$. The critical temperature rise $\Delta T$ of the functionally graded beam is contained in the thermal axial forces. It can be obtained by inverse solving $\theta=\frac{N^{T} l^{2}}{D}$.

Tab.1 the Critical Temperature Rise of the FGM Beam

\begin{tabular}{cccccccc}
\hline order & 1 & 2 & 3 & order & 1 & 2 & 3 \\
\hline$\theta_{n}$ & 20.19 & 59.67 & 118.90 & $\theta_{n}$ & 20.19 & 59.67 & 118.90 \\
\hline $\mathrm{SiC}$ & 249.76 & 738.15 & 1470.86 & $k=5$ & 110.69 & 327.13 & 651.86 \\
$k=0.5$ & 157.37 & 465.09 & 926.76 & $k=10$ & 101.55 & 300.13 & 598.05 \\
$k=1$ & 137.41 & 406.11 & 809.23 & $k=100$ & 85.87 & 253.80 & 505.73 \\
$k=2$ & 123.80 & 365.89 & 729.08 & $\mathrm{Ni}$ & 83.61 & 247.11 & 492.41 \\
\hline
\end{tabular}

Table 1 shows the critical temperature rise of FGM beam. It can be found that the critical temperature rise significantly increase with the increasing of the order of modes. With volume fraction index $k$ of material constituents increasing, the critical temperature rise decrease. However, with the increasing of $k$, the ability of withstanding thermal shock decreases.

\section{Summary}

The dynamic buckling characteristics of functionally graded beam under the thermal shock are studied. The solving process for dynamic buckling of the functionally graded beam subjected to thermal shock is 
established by using symplectic method. The problem is finally reduced to zero-eigenvalue problem in the symplectic space. The buckling mode and critical thermal axial forces are obtained through bifurcation condition, and the critical temperature rise of the functionally graded beam is obtained by inverse solution. The results show that the critical temperature rise significantly increases with the increasing of the order of modes. With volume fraction index $\mathrm{k}$ of material constituents increasing, the critical temperature rise decrease.

\section{Acknowledgement}

This work was financially supported by the National Natural Science Foundation of China (Nos.11262010,11272278) and the Fundamental Research Funds for the Universities of Gansu. The authors gratefully acknowledge all of the support.

\section{References}

[1]P. J. Chen, S. H. Chen, Progress in contact mechanics of a type of functional gradient materials [J], Advances in Mechanics. 44(2014) 1-25.

[2]R. Javaheri, M. R. Eslami, Thermal buckling of functionally graded plates [J], AIAA Journal. 40 (2002) 162-169.

[3]L. S. Ma, T. J. Wang, Relationships between the solutions of axisymmetric bending and buckling of functionally graded circular plates based on the third-order plate theory and the classical solutions for isotropic circular plates [J], International Journal of Solids and Structures. 41(2004) 85-101.

[4]B. Mirzavand, M. R. Eslami, M. Shakeri, Dynamic thermal postbuckling analysis of piezoelectric functionally graded cylindrical shells [J], Journal of Thermal Stresses. 33 (2010) 646-660.

[5]B. Mirzavand, M. R. Eslami, J. N. Reddy, Dynamic thermal postbuckling analysis of shear deformable piezoelectric FGM cylindrical shells [J], Journal of Thermal Stresses. 36 (2013) 189-206.

[6]M. Shariyat, Dynamic thermal buckling of suddenly heated temperature-dependent FGM cylindrical shells under combined axial compression and external pressure [J], International Journal of Solids and Structures. 45 (2008) 2598-2612.

[7]S. R. Li, L.L. Fan, Dynamic responses of functionally graded material beams under thermal shock [J], Journal of Vibration Engineering. 22 (2009) 371-378. 